\title{
Lues maligna bei unklarer Immundefizienz
}

\section{Lues maligna Associated with an Unknown Immundeficiency}

Autoren

Institut
T. Christoph, I. Feldmann-Böddeker

Hautklinik (Chefärztin: Dr. med. I. Feldmann-Böddeker), DRK-Krankenhaus Chemnitz-Rabenstein

\section{Bibliografie}

DOI $10.1055 / \mathrm{s}-2007-966902$

Akt Dermatol 2007; 33:

371-372 @ Georg Thieme

Verlag KG Stuttgart · New York ISSN 0340-2541

Korrespondenzadresse

OA Thomas Christoph

Hautklinik, DRK-Krankenhaus

Chemnitz-Rabenstein

Unritzstr. 23

09117 Chemnitz

Christoph.Thomas@

drk-chemnitz.de

\section{Zusammenfassung \\ $\nabla$}

Vorgestellt wird ein Patient mit einem generalisierten makulopapulösen, teils nekrotisierenden Exanthem, welches sich in der weiteren Diagnostik als Ausdruck einer Lues maligna herausstellt. Dieser liegt eine Immunschwäche zugrunde, die aufgrund eines nicht sehr kooperativen Patienten nicht weiter abgeklärt werden konnte.

\section{Einleitung \\ $\nabla$}

Die Lues maligna ist eine sehr seltene foudroyante Verlaufsform der Lues acquisita. Klinisch findet sie ihren Ausdruck als ulzerierendes Exanthem. Sie wird häufiger bei Patienten mit einer Abwehrschwäche (Immundefizienz) beobachtet. Insbesondere muss dabei auch an eine HIV-Infektion gedacht werden. Das Allgemeinbefinden ist ebenfalls oft erheblich beeinträchtigt. Unbehandelt kann die Lues maligna tödlich verlaufen.

\section{Anamnese \\ $\nabla$}

Die stationäre Aufnahme des 42-jährigen Patienten erfolgte aufgrund von generalisierten erythematösen Hautveränderungen nach Varizelleninfektion Ende Januar 2007. Frühere Hautkrankheiten seien nicht bekannt. Allgemeinsymptome wie Abgeschlagenheit, Fieber, Nachtschweiß, Gelenkbeschwerden oder Gewichtsabnahme negiert der Patient. Allerdings zeigte sich bei der stationären Aufnahme ein kachektischer Habitus.

\section{Aufnahmebefund \\ $\nabla$}

Bei Aufnahme befanden sich am gesamten Integument erythematöse, unscharf begrenzte, infiltrierte Makulae und Papeln, zum Teil konfluierend, zum Teil mäßig schuppend bzw. hyperkeratotisch belegt. Vor allem an den Oberschenkeln streckseitig wiesen die Hautveränderungen auch mittig aufsitzend dicke Krusten auf ( $\bullet$ Abb. 1). Finger- und Zehennägel zeigten zum Teil Onychodystrophien. An der Glans penis fanden sich weißliche abstreifbare Beläge. Vergrößerte Lymphknoten waren cervical bds., axillär rechts sowie inguinal bds. zu tasten.

Im Verlauf des stationären Aufenthaltes kam es zu zentralen Ulzerationen einiger Papeln (Rupia syphilitica) v.a. im Brustbereich. Im Gesicht waren eher konfluierende infiltrierte Erytheme zu sehen.

\section{Befunde diagnostischer Untersuchungen \\ $\nabla$}

Im Blutbild fiel zunächst eine deutliche Anämie (Hb 5,4 mmol/l, Hk 0,26, Erythrozyten 3,36 Tpt/l) auf. Die Lues-Serologie zeigte hochtitrige Befunde (VDRL-Test 1:128, Cardiolipin-KBR 1:10240, FTA-Abs-IgG 1:81920, FTA-Abs-IgM 1:640). Eine entsprechend weiterführende STD-Diagnostik (Gonorrhoe, Chlamydien, Hepatitis) zeigte unauffällige Befunde. Allerdings lehnte der Patient die Durchführung eines HIV-Testes ab.

Im Zellulären Immunstatus (T-Helfer/T-Suppressorzellverh.: 0,1; T-Helferzellen (relativ): 9\%,

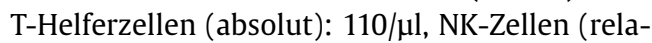

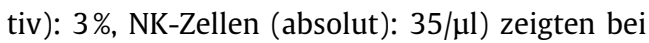
absolut normalen Lymphozyten die Absolutwerte der B- und NK-Zellen ein deutliches Defizit, die der Gesamt-T-Zellen lagen im Normbereich, die Helfer/Suppressor-Ratio erschien sehr niedrig aufgrund der stark vermehrten Helferzellen. Die 


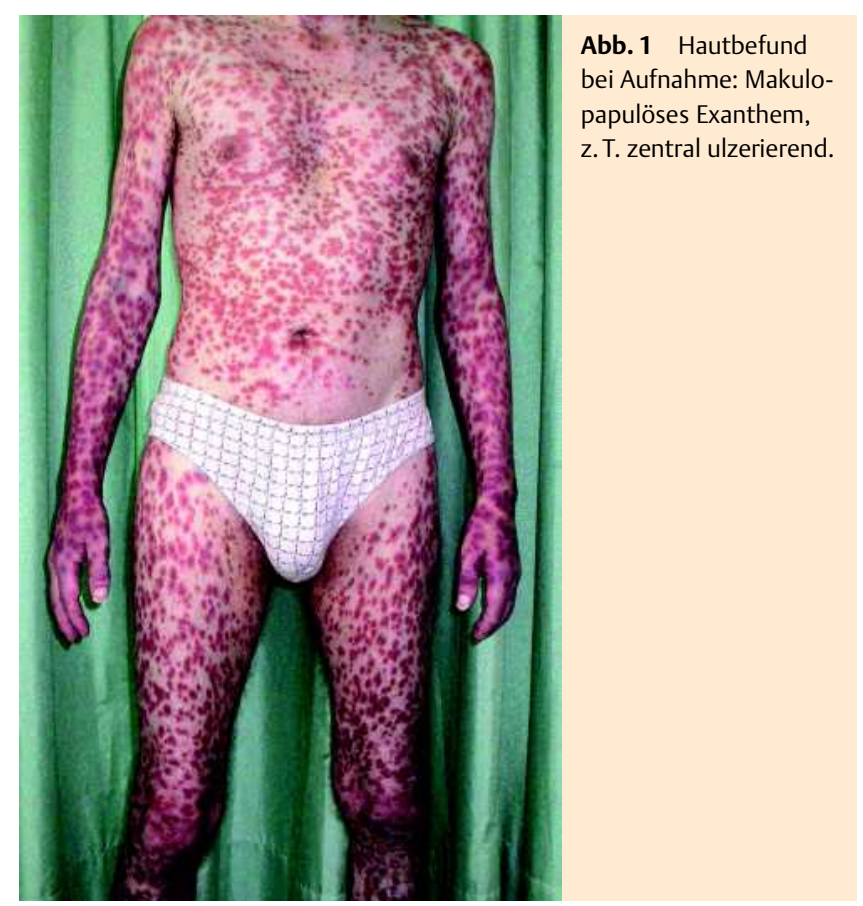

Veränderungen könnten mit HIV und anderen Virusinfektionen vereinbar sein, ein Immundefekt kann ebenfalls nicht ausgeschlossen werden.

Die Leberwerte waren deutlich erhöht (ALAT 7,66 $\mu \mathrm{mol} / \mathrm{sl}$, ASAT 1,36 $\mu \mathrm{mol} / \mathrm{sl}$, GGT 2,98 $\mu \mathrm{mol} / \mathrm{sl}$ ). Auch der Quick-Wert von $43 \%$ ohne orale Antikoagulation ließ an eine Leberbeteiligung der Lues denken. Eine Hepatitis konnte serologisch ausgeschlossen werden. Sonographisch (Abdomen und Lymphknotenstationen) konnte eine generalisierte Lymphknotenschwellung gesichert werden; sonst wurden keine Auffälligkeiten gefunden.

Weiterführende Diagnostik zum Ausschluss opportunistischer Infektionen (EBV, Toxoplasmose, Cytomegalie-Serologie) waren bis auf einen positiven Candidaabstrich im Mundbereich unauffällig.

Die proktologische und rektoskopische Untersuchung war unauffällig, ebenso eine Röntgenaufnahme des Thorax. Der Haemoccult-Test zum Ausschluss von Blut im Stuhl war mehrfach negativ.

Die Histologie einer diagnostischen Exzision der Haut zeigte ein dichtes Infiltrat aus Lymphozyten, Histiozyten und Plasmazellen im dermalen Stroma. Die Histologie war somit mit einer Lues vereinbar.

Leider lehnte der Patient trotz mehrmaliger ausführlicher Gespräche einen HIV-Test ab. Auch einer Anamneseerhebung hinsichtlich seines Sexualverhaltens blieb er verschlossen.

\section{Therapie und Verlauf $\nabla$}

Zunächst dachten wir aufgrund der infiltrierten Erytheme im Gesichtsbereich neben der Lues auch differentialdiagnostisch an ein Lymphom. Die hochtitrige Lues-Serologie und der histolo- gische Befund der Hautprobe bestätigten allerdings die Verdachtsdiagnose einer Lues.

Ein Aortenaneurysma konnte mittels Röntgen-Thorax ausgeschlossen werden. Eine entsprechende Penicillin-G-Therapie ( 3 x 10 Mio IE i.v) wurde eingeleitet. Bis zur Diagnosestellung waren zunehmende zentrale Nekrosen der Papeln v. a. im Oberkörperbereich zu verzeichnen. Da der Patient auch einer Liquordiagnostik ablehnend gegenüberstand, führten wir diese Antibiose für 4 Wochen durch.

Während der Therapie konnte zunächst ein Aufhalten der Nekrosebildung festgestellt werden. Das Exanthem blasste nur sehr langsam ab, nach 4 Wochen Therapie war es jedoch deutlich blasser als im Ausgangsbefund.

\section{Diskussion \\ $\nabla$}

Die Lues maligna ist eine seltene foudoryante Verlaufsform der Lues bei geschwächter Abwehrlage. Klinisches Kennzeichen ist das ulzerierende Exanthem. Die Ulzerationen sind von schüsselförmigen austernschalenartigen Borken (Rupia syphilitica) bedeckt und besitzen einen weichen Rand. Die Lues maligna verläuft als schweres konsumierendes Krankheitsbild mit deutlich eingeschränktem Allgemeinbefinden des Patienten (Fieber, Gewichtsverlust, Leistungsverlust). Dies lässt an eine Immunschwäche denken. Auch eine Beteiligung der inneren Organe (Hepatitis, Nephritis) ist möglich.

Im vorgestellten Fall bleibt sowohl der Infektionszeitpunkt als auch der Infektionsweg aufgrund des verschlossenen Patienten ungeklärt. Auch die klinischen und paraklinischen Hinweiszeichen auf eine HIV-Infektion konnten serologisch nicht weiter verifiziert werden. Trotz mehrfacher Gespräche war der Patient zu einem HIV-Test und zum Ausschluss einer Neurosyphilis mittels Liquorpunktion nicht bereit.

Die Therapie des Patienten wurde deshalb mit Penicillin G $3 \times 10$ Mio IE/d über 4 Wochen durchgeführt.

\section{Abstract}

\section{Lues maligna Associated with an Unknown Immundeficiency} $\nabla$

We present a patient with an exanthema with partially ulcerated papules. The diagnosis of a lues maligna is assumed to be associated with an unknown immundeficiency. The patient refused any further investigations to elucidate the cause of the immundeficiency.

\section{Literatur}

1 Hamouda O, Marcus U. Syphilis auf dem Vormarsch. Hautarzt 2005; 56: $124-132$

2 Körber A, Dissemond J, Hillen $U$ et al. HIV-positiver Patient mit multiplen Ulzerationen. Hautarzt 2003; 54: 1098 - 1102

3 Leitlinien der deutschen STD-Gesellschaft, Arbeitsgemeinschaft der wissenschaftlichen, medizinischen Fachgesellschaften Nr. 059/002. AWMF-online, Stand 2006 